International Journal of Applied Mathematics

Volume 32 No. $3 \quad 2019,443-449$

ISSN: $1311-1728$ (printed version); ISSN: 1314-8060 (on-line version)

doi: http://dx.doi.org/10.12732/ijam.v32i3.6

\title{
ON A CLASS OF SOLUTIONS FOR THE HYPERBOLIC DIOPHANTINE EQUATION
}

\author{
J. Kannan ${ }^{1 \S}$, Manju Somanath ${ }^{2}$, K. Raja ${ }^{3}$ \\ ${ }^{1}$ Ayya Nadar Janaki Ammal College \\ Sivakasi, 626 124, INDIA \\ ${ }^{2,3}$ National College (Autonomous) \\ Trichy, 620 001, INDIA
}

\begin{abstract}
Let $\mu \geq 2$ be a natural number. In this paper, we find all the solutions of the Hyperbolic Diophantine equations $D: x^{2}-\left(\mu^{2}-\mu\right) y^{2}-(4 \mu+$ $2) x+\left(6 \mu^{2}-6 \mu\right) y-(5 \mu-13) \mu=0$ over $\mathbb{Z}$. We also derive some recurrence relations on the integer solutions $\left(x_{n}, y_{n}\right)$ of $D$.
\end{abstract}

AMS Subject Classification: 11D09

Key Words: Diophantine equation, Pell equation, linear transformation, continued fraction

\section{Introduction}

A Diophantine equation is a polynomial equation $P\left(x_{1}, \cdots, x_{n}\right)=0$, where the polynomial $P$ has integral coefficients and one is interested in solutions for which all the unknowns take integer values. Diophantine equations are rich in variety. Two-variable Diophantine equation have been a subject of great interest, $[4,5]$. Their theory constitutes one of the most beautiful, most elaborate part of mathematics, which nevertheless still keeps some of its secrets for the next generation of researchers.

Received: January 16, 2019

(C) 2019 Academic Publications

${ }^{\S}$ Correspondence author 
In this paper, we investigate positive integral solutions of the Diophantine equation $x^{2}-\left(\mu^{2}-\mu\right) y^{2}-(4 \mu+2) x+\left(6 \mu^{2}-6 \mu\right) y-(5 \mu-13) \mu=0$ which is transformed into a Pell's equation and is solved by various methods.

\section{Preliminaries}

The Diophantine quadratic equation

$$
a x^{2}+b x y+c y^{2}+d x+e y+f=0
$$

with integral coefficient $a, b, c, d, e, f$ reduces in its main case to a Pell type equation. We will sketch the general method of reduction. Equation (1) represents a conic in the Cartesian plane, so solving (1) in integers means finding all lattice points situated on this conic. We will solve equation (1) as $\Delta=b^{2}-4 a c$. When $\Delta<0$, then conic defined by (1) is an ellipse, and in this case the give equation has only a finite number of solutions. When $\Delta=0$, the conic given by (1) is a parabola. If $2 a c-b d=0$, then equation (1) becomes $(2 a x+b y+d)^{2}=d 2-4 q f$, which is not difficult to solve. In the case $2 a e-b d \neq 0$, by performing the substitutions $X=2 a x+b y+d$ and $Y=(4 a c-2 b d) y+4 a f-d^{2}$, equation (1) reduces to $X^{2}+Y=0$, which is easy to solve. The most interesting case is $\Delta>0$, when the conic defined by (1) is a hyperbola, [7]. Using a sequence of substitutions, equation (1) reduces to the general Pell-type equation

$$
X^{2}-D Y^{2}=N
$$

$$
\begin{gathered}
\text { 3. The Diophantine Equation } \\
x^{2}-\left(\mu^{2}-\mu\right) y^{2}-(4 \mu+2) x+\left(6 \mu^{2}-6 \mu\right) y-(5 \mu-13) \mu=0
\end{gathered}
$$

In $[1,2,3]$, we consider some specific Pell (also Diophantine) equations and their integer solutions. In this present paper, we consider the integer solutions of Diophantine equation

$$
D: x^{2}-\left(\mu^{2}-\mu\right) y^{2}-(4 \mu+2) x+\left(6 \mu^{2}-6 \mu\right) y-(5 \mu-13) \mu=0
$$

over $\mathbb{Z}$, where $\mu \geq 2$ is an integer. It is not easy to solve and find the nature and properties of the solutions of (3). So we apply a linear transformation $T$ to (3) to transfer to a simpler form for which we can determine the integral solutions. 
Let

$$
T:=\left\{\begin{array}{l}
x=u+h \\
y=v+k
\end{array}\right.
$$

be the transformation where $h, k \in \mathbf{Z}$. Applying $T$ to $D$, we get

$$
\left\{\begin{aligned}
T(D)=\tilde{D}: & (u+h)^{2}-\left(\mu^{2}-\mu\right)(v+k)^{2}-(4 \mu+2)(u+h) \\
& +\left(6 \mu^{2}-6 \mu\right)(v+k)-(5 \mu-13) \mu=0
\end{aligned}\right.
$$

equating the coefficients of $u$ and $v$ to zero, we get $h=2 \mu+1$ and $k=3$. Hence for $x=u+2 \mu+1$ and $y=v+3$, we have the Diophantine equation

$$
\tilde{D}: u^{2}-\left(\mu^{2}-\mu\right) v^{2}=1
$$

which is Pell equation. Now we try to find all integer solutions $\left(u_{n}, v_{n}\right)$ of $\tilde{D}$ and then we can re transfer all results from $\tilde{D}$ to $D$ by using the inverse of $T$.

Theorem 1. Let $\tilde{D}$ be the Diophantine equation in (6). Then

1. The continued fraction expansion of $\sqrt{\mu^{2}-\mu}$ is

$$
\sqrt{\mu^{2}-\mu}=\left\{\begin{array}{l}
{[1 ; \overline{2}], \text { if } \mu=2} \\
{[\mu-1 ; \overline{2,2 \mu-2}], \text { if } \mu>2}
\end{array}\right.
$$

2. The fundamental solution of $\tilde{D}$ is $\left(u_{1}, v_{1}\right)=(2 \mu-1,2)$.

3. Define the sequence $\left\{\left(u_{n}, v_{n}\right)\right\}$, where

$$
\left(\begin{array}{l}
u_{n} \\
v_{n}
\end{array}\right)=\left(\begin{array}{cc}
2 \mu-1 & 2 \mu^{2}-2 \mu \\
2 & 2 \mu-1
\end{array}\right)^{n}\left(\begin{array}{l}
1 \\
0
\end{array}\right)
$$

for $n \geq 1$. Then $\left(u_{n}, v_{n}\right)$ is a solution of $\tilde{D}$.

4. The solutions $\left(u_{n}, v_{n}\right)$ satisfy $u_{n}=(2 \mu-1) u_{n-1}+\left(2 \mu^{2}-2 \mu\right) v_{n-1}$ and $v_{n}=2 u_{n-1}+(2 \mu-1) v_{n-1}$ for $n \geq 2$.

5. The solutions $\left(u_{n}, v_{n}\right)$ satisfy the recurrence relations $u_{n}=(4 \mu-3)\left(u_{n-1}+u_{n-2}\right)-u_{n-3}$ and
$v_{n}=(4 \mu-3)\left(v_{n-1}+v_{n-2}\right)-v_{n-3}$ for $n \geq 4$.

6. The $n-t h$ solution $\left(u_{n}, v_{n}\right)$ can be given by

$$
\frac{u_{n}}{v_{n}}=[\mu-1 ; 2, \underbrace{2 \mu-2, \cdots, 2,2 \mu-2}, 2],
$$

for $n \geq 1$. 
We saw as above that the Diophantine equation $D$ could be transformed into the Diophantine equation $\tilde{D}$ via the transformation $T$. Also we showed that $x=u+2 \mu+1$ and $y=v+3$. so we can re transfer all results from $\tilde{D}$ to $D$ by using the inverse of $T$. Thus we can give the following main theorem.

\section{Main Results}

Theorem 2. 1. The fundamental (minimal) solution of $D$ is $\left(x_{1}, y_{1}\right)=$ $(4 \mu, 5)$.

2. Define the sequence $\left\{\left(x_{n}, y_{n}\right)\right\}_{n \geq 1}=\left\{\left(u_{n}+2 \mu+1, v_{n}+3\right)\right\}$, where $\left(u_{n}, v_{n}\right)$ is defined in (7). Then $\left(x_{n}, y_{n}\right)$ is a solution of $D$. So it has infinitely many integer solutions $\left(x_{n}, y_{n}\right) \in \mathbb{Z} \times \mathbb{Z}$.

3. The solutions $\left(x_{n}, y_{n}\right)$ satisfy, for $n \geq 2$

$$
\begin{aligned}
& x_{n}=(2 \mu-1) x_{n-1}+\left(2 \mu^{2}-2 \mu\right) y_{n-1}-10 \mu^{2}+8 \mu+2 . \\
& y_{n}=2 x_{n-1}+(2 \mu-1) y_{n-1}-10 \mu+4 .
\end{aligned}
$$

4. The solutions $\left(x_{n}, y_{n}\right)$ satisfy the recurrence relations,

for $n \geq 4$,

$$
\begin{aligned}
& x_{n}=(4 \mu-3)\left(x_{n-1}+x_{n-2}\right)-x_{n-3}-16 \mu^{2}+8 \mu+8, \\
& y_{n}=(4 \mu-3)\left(y_{n-1}+y_{n-2}\right)-y_{n-3}-24 \mu+24 .
\end{aligned}
$$

Proof. 1. It is easily seen that $\left(x_{1}, y_{1}\right)=(4 \mu, 5)$ is the fundamental solution of $D$ since $(4 \mu)^{2}-\left(\mu^{2}-\mu\right)(5)^{2}-(4 \mu+2)(4 \mu)+\left(6 \mu^{2}-6 \mu\right)(5)-$ $(5 \mu-13) \mu=0$.

2. We prove it by Mathematical induction. Let $n=1$.

Then $\left(x_{1}, y_{1}\right)=\left(u_{1}+2 \mu+1, v_{1}+3\right)=(4 \mu, 5)$ which is the fundamental solution and so is solution of $D$. Let us assume that the Diophantine equation in (1) is satisfied for $n-1$, that is $x_{n-1}^{2}-\left(\mu^{2}-\mu\right) y_{n-1}^{2}-(4 \mu+$ 2) $x_{n-1}+\left(6 \mu^{2}-6 \mu\right) y_{n-1}-(5 \mu-13) \mu=0$. We want to show that this equation in (1) is satisfied for $n$.

$$
\begin{aligned}
x^{2}-\left(\mu^{2}-\mu\right) y^{2}- & (4 \mu+2) x+\left(6 \mu^{2}-6 \mu\right) y-(5 \mu-13) \mu \\
& =\left(u_{n}+2 \mu+1\right)^{2}-\left(\mu^{2}-\mu\right)\left(v_{n}+3\right)^{2} \\
& -(4 \mu+2)\left(u_{n}+2 \mu+1\right)
\end{aligned}
$$




$$
\begin{aligned}
& +\left(6 \mu^{2}-6 \mu\right)\left(v_{n}+3\right)-5(\mu-13) \mu \\
& =u_{n}^{2}-\left(\mu^{2}-\mu\right) v_{n}^{2}-1 \\
& =0\left(u_{n}, v_{n} \text { solution of } \tilde{D}\right) .
\end{aligned}
$$

So, $\left(x_{n}, z_{n}\right)=\left(u_{n}+2 \mu+1, v_{n}+5\right)$.

3. From (12), $u_{n}=(2 \mu-1) u_{n-1}+\left(2 \mu^{2}-2 \mu\right) v_{n-1}$.

Adding $2 \mu+1$ on both sides,

$\left(u_{n}+2 \mu+1\right)=(2 \mu-1) u_{n-1}+\left(2 \mu^{2}-2 \mu\right) v_{n-1}+2 \mu+1$

we know that $x_{n}=u_{n}+2 \mu+1$ and $y_{n}=v_{n}+3$.

Therefore, $u_{n}=x_{n}-(2 \mu+1)$ and $v_{n}=y_{n}-3$,

$$
\begin{aligned}
\left(u_{n}+2 \mu+1\right) & =(2 \mu-1) u_{n-1}+\left(2 \mu^{2}-2 \mu\right) v_{n-1}+2 \mu+1 \\
x_{n} & =(2 \mu-1)\left(x_{n-1}-(2 \mu+1)\right) \\
& +\left(2 \mu^{2}-2 \mu\right)\left(y_{n-1}-3\right)+2 \mu+1,
\end{aligned}
$$

and we get,

$$
x_{n}=(2 \mu-1) x_{n-1}+\left(2 \mu^{2}-2 \mu\right) y_{n-1}-10 \mu^{2}+8 \mu+2 .
$$

Similarly,

$$
y_{n}=2 x_{n-1}+(2 \mu-1) y_{n-1}-10 \mu+4
$$

4. We prove that $x_{n}$ satisfy the recurrence relation. For $n=4$, we get $x_{1}=4 \mu, x_{2}=8 \mu^{2}-6 \mu+2, x_{3}=32 \mu^{3}-48 \mu^{2}+20 \mu, x_{4}=128 \mu^{4}-256 \mu^{3}+$ $160 \mu^{2}-30 \mu+2$. Hence

$$
\begin{aligned}
x_{4} & =(4 \mu-3)\left(x_{3}+x_{2}\right)-x_{1}-16 \mu^{2}+8 \mu+8 \\
& =128 \mu^{4}-256 \mu^{3}+160 \mu^{2}-30 \mu+2 .
\end{aligned}
$$

So $x_{4}=(4 \mu-3)\left(x_{3}+x_{2}\right)-x_{1}-16 \mu^{2}+8 \mu+8$ is satisfied for $n=4$.

Let us assume that this relation is satisfied of $n-1$, that is

$$
x_{n-1}=(4 \mu-3)\left(x_{n-2}+x_{n-3}\right)-x_{n-4}-16 \mu^{2}+8 \mu+8 .
$$

Then applying the previous assertion, (9) and (11), we conclude that $x_{n}=(4 \mu-3)\left(x_{n-1}+x_{n-2}\right)-x_{n-3}-16 \mu^{2}+8 \mu+8$, for $n \geq 4$.

Similarly, we prove that $y_{n}$ satisfies the recurrence relation. For $n=4$, we get $y_{1}=5, y_{2}=8 \mu-1, y_{3}=32 \mu^{2}-32 \mu+9, y_{4}=128 \mu^{3}-192 \mu^{2}+80 \mu^{2}-5$. Hence

$$
y_{4}=(4 \mu-3)\left(y_{3}+y_{2}\right)-y_{1}-24 \mu+24
$$




$$
=128 \mu^{3}-192 \mu^{2}+80 \mu-5 .
$$

So $y_{4}=(4 \mu-3)\left(y_{3}+y_{2}\right)-y_{1}-24 \mu+24$ is satisfied for $n=4$. Let us assume that this relation is satisfied for $n-1$, that is

$$
y_{n-1}=(4 \mu-3)\left(y_{n-2}+y_{n-3}\right)-y_{n-4}-24 \mu+24 \mu .
$$

Then applying the previous assertion, (10) and (12), we conclude that $y_{n}=(4 \mu-3)\left(y_{n-1}+y_{n-2}\right)-y_{n-3}-24 \mu+24$, for $n \geq 4$.

\section{Conclusion}

Diophantine equations are rich in variety. There is no universal method for finding all possible solutions (if it exists) for Diophantine equations. The method looks to be simple but it is very difficult for reaching the solutions.

\section{References}

[1] I. Niven, H.S. Zuckerman, H.L. Montgometry, An Introduction to the Theory of Numbers, Fifth Edition, John Wiley and Sons, Inc., New York (1991).

[2] D.M. Burton, Elementary Number Theory, Tata McGraw-Hill Ed. (2012).

[3] L.J. Mordell, Diophantine Equations, Academic Press, New York (1969).

[4] M. Somanath, J. Kannan, K. Raja and V. Sangeetha, On the integer solutions of the Pell equation $x^{2}=17 y^{2}-19^{t}$, JP Journal of Applied Mathematics, 15, No 2 (2017), 81-88.

[5] M. Somanath, J. Kannan, On a class of solutions for a Diophantine equation of second degree, Intern. J. of Pure and Applied Math., Special Issue, 117, No 12 (2017), 55-62.

[6] D. Hensley, Continued Fractions, World Scientific Publishing, Hackensack, N.J. (2006).

[7] T. Andreescu, An Introduction to Diophantine Equations, A Problem Based Approach (2010). 
[8] J. Kannan, M. Somanath, K. Raja, Solutions of negative Pell equation involving twin prime, JP Journal of Algebra, Number Theory and Applications, 40, No 5 (2018), 869-874.

[9] M. Somanath, J. Kannan, K. Raja, Lattice points of an infinite cone $x^{2}+$ $y^{2}=\left(\alpha^{2 n}+\beta^{2 n}\right) z^{2}$, International J. of Mathematics Trends and Technology (IJMTT), 38, No 2 (2016), 95-98.

[10] M. Somanath, J. Kannan, K. Raja, Exponential Diophantine equation in two and three variables, Global J. of Pure and Applied Mathematics, 13, No 5 (2017), 128-132.

[11] M. Somanath, J. Kannan, On the positive integer solutions for a Diophantine equation, J. of Mathematics and Informatics, 10 (2017), 173-177.

[12] M. Somanath, J. Kannan, Congruum problem, Intern. J. of Pure and Applied Math. Sciences, 9, No 2 (2016), 123-131. 
